\title{
OKA'S HEFTUNGSLEMMA \\ AND THE LEVI PROBLEM FOR COMPLEX SPACES
}

\author{
BY \\ ALDO ANDREOTTI AND RAGHAVAN NARASIMHAN
}

Introduction. This paper has its origin in an analysis of $\mathrm{K}$. Oka's solution of the Levi problem for unramified domains over $C^{n}$ [11]. It consists of two parts. The first part contains a new proof of the following theorem [9].

THEOREM 1. Let $X$ be a complex space and $p$ a continuous sirongly pseudoconvex function on $X$ such that for any $\alpha>0$ the set

$$
\{x \in X \mid p(x)<\alpha\}
$$

is relatively compact in $X$. Then $X$ is a Stein space.

The proof given here is based on a refined version of the Heftungslemma of Oka [11] which we give in $\$ 2$, and avoids the consideration of completely continuous mappings between Fréchet spaces [4]. It also avoids a detour through the following result, which was required in the older proof.

$A$ holomorph-convex $K$-complete, space is a Stein space.

In the second part, we consider the question of the extent to which the assumption of strong convexity can be dropped. Theorem 1 becomes false if we merely drop the assumption of strong convexity as shown by an example of $\mathbf{H}$. Grauert (see [10a] where this example is given). In this direction we prove.

THEOREM 2. On a K-complete space, any relatively compact open set which is pseudoconvex with a globally defined boundary is a Stein space.

We next turn to Stein spaces and prove (Theorem 4, Corollary 1) that on a Stein space with isolated singularities, an open set which is locally a Stein space is also globally a Stein space.

This we obtain as a special case of a theorem on Stein spaces whose singularities are not necessarily isolated. The particular case when $X$ has no singularities has already been treated by Docquier-Grauert. We have used in the proof the following result (Theorem 5).

A complex space all of whose irreducible components are Stein spaces is itself a Stein space.

Received by the editors February 4, 1963. 
This is a special case of a theorem [10] which asserts that a complex space whose normalisation is a Stein space is itself a Stein space. However, we have given a direct proof for the case which we need.

In the final section $(\$ 6)$ we have collected together some observations. We show that for complex manifolds, the statement that a holomorph-convex, $K$-complete manifold is a Stein manifold can be deduced from a "vanishing theorem" on noncompact kähler manifolds [2] proved by Andreotti and Vesentini. Finally, we apply Theorem 1 to the proof of the following two facts $[14 ; 15]$.

An unramified covering manifold of a Stein open set in $C^{n}$ is a Stein manifold. A holomorphic vector bundle over a Stein manifold is itself a Stein manifold.

1. Preliminaries. All complex spaces considered are supposed countable at infinity.

Let $X$ be a complex space. We say $X$ is holomorph-convex if for any infinite discrete set $E \subset X$, there is a holomorphic function $f$ on $X$ for which $f(E)$ is unbounded. A holomorph-convex space $X$ for which the following two properties hold is called a Stein space: (i) if $x \neq y$ are points of $X$, there is a holomorphic function $f$ on $X$ with $f(x) \neq f(y)$; (ii) for any $x \in X$, there exist $f_{1}, \cdots, f_{k}$ holomorphic on $X$ which map a neighborhood of $x$ isomorphically onto an analytic set in an open set in $C^{k}$.

A complex space $X$ is called $K$-complete if for every $x_{0} \in X$ there is a holomorphic map $f$ of $X$ into $C^{p}, p=p\left(x_{0}\right)$, such that $x_{0}$ is an isolated point of $f^{-1} f\left(x_{0}\right)$.

It is known [Grauert, Math. Ann. 129 (1955), 233-259] that a pure dimensional space $X$ of dimension $n$ is $K$-complete if and only if $X$ can be realised as a ramified domain over $C^{n}$, but we will not require this theorem.

We refer to $[9, \S \S 1,2]$ for the definition and elementary properties of Runge domains on Stein spaces and pseudoconvex and strongly pseudoconvex functions on complex spaces (called there convex and strongly convex functions respectively). We will use frequently the following result without explicit mention (see [15]).

(1.1) The union of an increasing sequence of Stein spaces, each of which is a Runge open subset of the succeeding one, is itself a Stein space.

The following theorem is proved in [9, p. 211].

(1.2) Let $X$ be a Stein space and $p$ a continuous pseudoconvex function on $X$. Then the set

$$
X_{0}=\{x \in X \mid p(x)<0\}
$$

is Runge in $X$. (In particular $X_{0}$ is a Stein space.)

We remark that the proof of (1.2) was based on an argument, which gives the following result without any change whatsoever. 
(1.3) Let $X$ be a Stein space and $K$ a compact subset of $X$. Let $\hat{K}$ be the set $\left\{x \in X|| f(x)\left|\leqq \sup _{y \in K}\right| f(y) \mid\right.$ for all $f$ holomorphic on $\left.X\right\}$. Let $x_{0} \in \hat{K}-K$. Let $U$ be a neighborhood of $x_{0}$ in $X$ and $f(x, t)$ a continuous function for $x \in U, 0 \leqq t \leqq 1$ which is holomorphic in $U$ for any $t$. Set $\sigma_{t}=\{x \in U \mid f(x, t)=0\}$, $C=\bigcup_{0 \leqq t \leqq 1} \sigma_{t}$.

Suppose that there exists a neighborhood $V$ of $\hat{K}$ such that $C \cap V$ is closed in $V$ while $K \cap C=\varnothing$ and $x_{0} \in \sigma_{0}$. Then there exist arbitrarily small $t>0$ such that $\sigma_{t} \cap \hat{K} \neq \varnothing\left({ }^{1}\right)$.

From this result we deduce the following one.

(1.4) Let $D$ be an open subset of the Stein space X. Suppose that $\partial D(=\bar{D}-D)$ has a neighborhood $U$ in which there exists a holomorphic function $g$ such that $C=\{u \in U \mid \operatorname{Re} g(u)=0\}$ is a closed subset of $X$ such that $\partial D \subset C, C \cap D=\varnothing$. Then $D$ is a Runge domain in $X$ (in particular, $D$ is a Stein space).

Proof of (1.4). Iet $K$ be a compact subset of $D$ and $\hat{K}$ its envelope in $X$ (i.e., the set of $x \in X$ where $|f(x)| \leqq \sup _{y \in K}|f(y)|$ for all $f$ holomorphic on $X$ ). We assert that $\hat{K} \cap C=\varnothing$. Suppose that, on the contrary, $C_{1}=\hat{K} \cap C \neq \varnothing$ and let $s=\sup _{x \in C_{1}} \operatorname{Im} g(x)$. Put $f(x, t)=g(x)-i s-i t$ in $U, 0 \leqq t \leqq 1$, $\sigma_{t}=\{x \in U \mid f(x, t)=0\}$. Then $\sigma_{t} \subset C$, so that $\bigcup \sigma_{t}$ is a closed subset of $C$ (hence of $X$ ) which does not meet $K \subset D$. Moreover, $\sigma_{t} \cap \hat{K}=\varnothing$ for $t>0$. Hence, by (1.3), we have $\sigma_{0} \cap \hat{K}=\varnothing$ which is false.

Thus $\hat{K} \cap \partial D=\varnothing$. Since every connected component of $\hat{K}$ meets $K[8, \mathrm{p}$. 919], it follows that $\hat{K} \subset D$. q.e.d.

We will need the following result (see proof of statement (2) on p. 334 in Grauert [5]).

(1.5) Let $p$ be a $C^{\infty}$ strongly pseudoconvex function in an open set $G \subset C^{n}, K \subset G$ a compact set. Then there exist numbers $r, \delta>0$ such that, for $z \in K$, the ball $H$ of radius $2 r$ about $z$ lies in $G$, while there exists in $H$ a holomorphic function $f$ such that

$$
\{x \in H \mid \operatorname{Re} f(x)=0\} \cap\{y \in G \mid p(y) \leqq p(z)\}=\{z\}
$$

and, if $x \in H$ satisfies $\operatorname{Re} f(x)=0,|x-z|=r$, then we have $p(x) \geqq p(z)+\delta$.

We may suppose also that $\operatorname{Re} f(y)<0^{\prime}$ if $p(y)<p(z)$.

\section{2. "Heftungslemmata."}

(2.1) First Heftungslemma. Let $X$ be a complex space, let $h_{1}, \cdots, h_{k}$ be holomorphic functions on $X$ and $g$ a holomorphic function on $X$ which is nowhere zero. We consider the sets:

$$
Y=\left\{x \in X|\quad| h_{i}(x) \mid<1 \text { for } 1 \leqq i \leqq k\right\}
$$

and, for every $\delta>0$,

(1) Essentially (1.3) is proved by G. Stolzenberg [16] for compact sets in $C^{n}$. Stolzenberg also remarked that the proof of $(1.3)$ becomes much simpler if $f(x, t)$ is supposed, in addition, to be analytic in $x$ and $t$. This case is sufficient for the results that we need in this paper. 
We have

$$
\begin{aligned}
& Y_{1}(\delta)=\left\{x \in Y|| g(x) \mid<e^{\delta}\right\}, \\
& Y_{2}(\delta)=\left\{x \in Y|| g(x) \mid>e^{-\delta}\right\} .
\end{aligned}
$$

We put

$$
Y=Y_{1}(\delta) \cup Y_{2}(\delta)
$$

$$
Y_{3}(\delta)=Y_{1}(\delta) \cap Y_{2}(\delta)
$$

LEMMA 1. Let us assume that for a suitable $\delta>0$

(i) $Y_{3}(\delta)$ is relatively compact in $X$;

(ii) the functions $h_{1}, \cdots, h_{k}, g$ give local coordinates and separate points of $Y_{3}(\delta)$.

Then for every holomorphic function $f$ on $Y_{3}(\delta)$ there exist holomorphic functions $f_{1}$ on $Y_{1}(\delta)$ and $f_{2}$ on $Y_{2}(\delta)$ such that on $Y_{3}(\delta)$ we have:

$$
f=f_{1}-f_{2} \text {. }
$$

Proof. Consider the map $\phi: X \rightarrow C^{k+1}$ given by

$$
x \rightarrow\left(g(x), h_{1}(x), \cdots, h_{k}(x)\right) .
$$

Consider the polyhedron in $C^{k+1}=C \times C^{k}$ :

$$
P=\left\{(z, w) \in C \times C^{k}\left|e^{-\delta}<\right| z \mid<e^{\delta},\|w\|<1\right\} .
$$

Then $\phi$ maps $Y_{3}(\delta)$ on a subset $A$ of $P$. By conditions (i), (ii) the map $\left.\phi\right|_{Y_{3}(\delta)}$ is proper. Hence $A$ is an analytic subset of $P$ isomorphic to $Y_{3}(\delta)$.

The function $f$ defines therefore a holomorphic function $\tilde{f}$ on $A$. Since $P$ is a product of $k+1$ open sets in the complex plane, it is a domain of holomorphy. Therefore we can find a holomorphic function $F$ on $P$ such that $\left.F\right|_{A}=\tilde{f}$.

Let $(z, w) \in P$. Then for every $0<\varepsilon<\varepsilon(z, w)$ (sufficiently small) we can write

$$
\begin{aligned}
F(z, w)= & \frac{1}{(2 \pi i)^{k+1}} \int_{|\zeta|=e^{\delta-\varepsilon}} \frac{d \zeta}{\zeta-z} \int_{\left|\eta_{1}\right|=1-\varepsilon} \frac{d \eta_{1}}{\eta_{1}-w_{1}} \cdots \int_{\left|\eta_{k}\right|=1-\varepsilon} \frac{F(\zeta, \eta)}{\eta_{k}-w_{k}} d \eta_{k} \\
& -\frac{1}{(2 \pi i)^{k+1}} \int_{|\zeta|=e^{-\delta}+\varepsilon} \frac{d \zeta}{\zeta-z} \int_{\left|\eta_{1}\right|=1-\varepsilon} \frac{d \eta_{1}}{\eta_{1}-w_{1}} \cdots \int_{\left|\eta_{k}\right|=1-\varepsilon} \frac{F(\zeta, \eta)}{\eta_{k}-w_{k}} d \eta_{k} .
\end{aligned}
$$

The first of these integrals defines a holomorphic function $F_{1}(z, w, \varepsilon)$ on the set $|z|<e^{\delta}-\varepsilon,\|w\|<1-\varepsilon$. If $\varepsilon^{\prime}<\varepsilon^{\prime \prime}$ the corresponding functions $F_{1}\left(z, w, \varepsilon^{\prime}\right)$ and $F_{1}\left(z, w, \varepsilon^{\prime \prime}\right)$ coincide in the common region of definition. Therefore we obtain a holomorphic function $F_{1}(z, w)$ defined on the set

$$
P_{1}=\left\{(z, w) \in C \times C^{k}|| z \mid<e^{\delta},\|w\|<1\right\} .
$$

Analogously from the second of the above integrals, we obtain a holomorphic function $F_{2}(z, w)$ on the set 


$$
P_{2}=\left\{(z, w) \in C \times C^{k}\left|e^{-\delta}<\right| z \mid,\|w\|<1\right\}
$$

and by the above expression of $F$ we have on $P=P_{1} \cap P_{2}$

$$
F=F_{1}-F_{2} \text {. }
$$

Since $Y_{1}(\delta)=\phi^{-1}\left(P_{1}\right), \quad Y_{2}(\delta)=\phi^{-1}\left(P_{2}\right)$ it is enough to take $f_{1}=F_{1} \circ \phi$, $f_{2}=F_{2} \circ \phi$ on $Y_{1}(\delta), Y_{2}(\delta)$ respectively to get the result.

Corollary. If $Y_{1}(\delta)$ and $Y_{2}(\delta)$ are Stein spaces we then have: $H^{1}(Y, \mathcal{O})=0$ where $\mathcal{O}$ is the sheaf of germs of holomorphic functions on $X$, and, for any coherent sheaf $\mathscr{F}$ on $Y, H^{i}(Y, \mathscr{F})=0$ if $i \geqq 2$.

(2.2) Second Heftungslemma. This is our version of the central idea of Oka [11]. The essential point is to replace the assumption that $g, h_{i}$ are globally defined in Lemma 1 above by more geometric conditions.

Let $g$ be a continuous function nowhere zero on the complex space $X$. We suppose that $g$ is holomorphic in the set

$$
X_{3}(\delta)=\left\{x \in X\left|e^{-\delta}<\right| g(x) \mid<e^{+\delta}\right\}
$$

for some $\delta>0$. For any $\rho<\delta$, we set

$$
X_{1}(\rho)=\left\{x \in X|| g(x) \mid<e^{+\rho}\right\}, \quad X_{2}(\rho)=\left\{x \in X|| g(x) \mid>e^{-\rho}\right\}
$$

We suppose that

(i) $X_{1}(\delta), X_{2}(\delta)$ are Stein spaces such that $X_{3}(\delta)$ is Runge in each of them.

Let $h_{1}, \cdots, h_{k}$ be holomorphic in $X_{3}\left(\delta^{\prime}\right)$ (where $\delta^{\prime}>\delta$ ) such that

(ii) there is $\varepsilon_{0}>0$ such that $\left|h_{i}(x)\right|<1-\varepsilon_{0}$ if $|g(x)|=e^{ \pm \delta}$.

For $0 \leqq \varepsilon \leqq \varepsilon_{0}, 0<\rho<\delta$, we set

$$
Y_{1}(\varepsilon, \rho)=\left[X_{1}(\delta)-X_{3}(\delta)\right] \cup\left\{x \in X_{3}(\delta)|| h_{i}(x)|<1-\varepsilon,| g(x) \mid<e^{+\rho}\right\}
$$

and similarly, replacing $X_{1}(\delta)$ by $X_{2}(\delta)$ and $\left\{|g|<e^{+\rho}\right\}$ by $\left\{|g|>e^{-\rho}\right\}$, we define the set $Y_{2}(\varepsilon, \rho)$. Clearly, because of (ii), $Y_{j}(\varepsilon, \rho)$ is an open set for $j=1,2$. Set $Y_{3}(\varepsilon, \rho)=Y_{1}(\varepsilon, \rho) \cap Y_{2}(\varepsilon, \rho)$ and $Y(\varepsilon)=Y_{1}(\varepsilon, \rho) \cup Y_{2}(\varepsilon, \rho)$. We suppose now that

(iii) for some $\rho_{0}, 0<\rho_{0}<\delta$, the set $Y_{3}\left(0, \rho_{0}\right)$ is relatively compact in $X$ and the functions $g, h_{1}, \cdots, h_{k}$ separate points and give local coordinates on it.

LEMMA 2. Under the assumptions (i), (ii), (iii) made above, if $f$ is a holomorphic function in $X_{3}(\delta)$, then for any $\rho<\rho_{0}$, and $0<\varepsilon<\varepsilon_{0}$, there exist holomorphic functions $f_{r}$ in $Y_{r}(\varepsilon, \rho)(r=1,2)$ such that

$$
f_{1}-f_{2}=f \text { in } Y_{3}(\varepsilon, \rho) \text {. }
$$

Moreover, for any compact sets $K_{r} \subset Y_{r}(\varepsilon, \rho)(r=1,2)$, there exist constants $M_{r}>0$, independent of $f$ such that

$$
\left\|f_{r}\right\|_{K_{r}} \leqq M_{r}\|f\|_{\dot{Y}_{3}(\varepsilon, \rho)}
$$


Proof. Let $\phi: X_{3}(\delta) \rightarrow C^{k+1}$ be defined by $\phi(x)=\left(g(x), h_{1}(x), \cdots, h_{k}(x)\right)$. Because of (iii), $\phi$ maps $Y_{3}\left(0, \rho_{0}\right)$ isomorphically onto an analytic set $A$ of the polyhedron $P_{0, \rho_{0}}$, where, for $0 \leqq \varepsilon<\varepsilon_{0}, 0<\rho \leqq \delta$, we set

$$
P_{\varepsilon, \rho}=\left\{(z, w) \in C^{k+1}\left|e^{-\rho}<\right| z\left|<e^{\rho},\right| w_{v} \mid<1-\varepsilon \text { for } 1 \leqq v \leqq k\right\} .
$$

By assumption (iii), the set $Y_{3}(\varepsilon, \rho), \rho \leqq \rho_{0}$, is relatively compact in $X_{3}(\delta)$, so that

$$
\|f\|_{Y_{3}(\varepsilon, \rho)}=\sup |f(y)| \text { for } y \in Y_{3}(\varepsilon, \rho)
$$

is finite.

Since $P_{\varepsilon, \rho}$ is a domain of holomorphy, for $\varepsilon<\varepsilon^{\prime}, \rho^{\prime}<\rho$ there exists $[6, \S 8]$ a constant $M=M\left(\varepsilon^{\prime}, \rho^{\prime}\right)>0$ independent of $f$, and a holomorphic function $F$ in $P_{\varepsilon, \rho}$, such that $F \circ \phi=f$ on $Y_{3}(\varepsilon, \rho)$, with

$$
\|F\|_{P_{\varepsilon^{\prime}, \rho},} \leqq M\|f\|_{Y_{3}(\varepsilon, \rho)} .
$$

Let $(z, w) \in P_{\varepsilon^{\prime}, \rho^{\prime}}$. Then we have

$$
F(z, w)=\frac{1}{(2 \pi i)^{k+1}} \int_{\left|\eta_{v}\right|=1-\varepsilon^{\prime}} \ldots \int\left[\int_{|\zeta|=e^{q^{\prime}}}-\int_{|\zeta|=e^{-q}}\right] \frac{F(\zeta, \eta)}{\left.(\zeta-z) \prod^{\left(\eta_{v}\right.}-w_{v}\right)} d \zeta d \eta_{1} \cdots d \eta_{k^{\prime}} .
$$

Hence if we set

$$
\begin{aligned}
\chi(x, \zeta, \eta) & =(2 \pi i)^{-k-1}(\zeta-g(x))^{-1} \prod\left(\eta_{v}-h_{v}(x)\right)^{-1}, \\
\Gamma_{1} & =\left\{(\zeta . \eta) \in C^{k+1}|| \zeta\left|=e^{\rho^{\prime}},\right| \eta_{v} \mid=1-\varepsilon^{\prime} \text { for } 1 \leqq v \leqq k\right\}, \\
\Gamma_{2} & =\left\{(\zeta, \eta) \in C^{k+1}|| \zeta\left|=e^{-\rho^{\prime}},\right| \eta_{v} \mid=1-\varepsilon^{\prime}, \text { for } 1 \leqq v \leqq k\right\},
\end{aligned}
$$

we have $f(x)=\left(\int_{\Gamma_{1}}-\int_{\Gamma_{2}}\right) \chi(x, \zeta, \eta) F(\zeta, \eta) d \zeta d \eta$, for $x \in Y_{3}\left(\varepsilon^{\prime}, \rho^{\prime}\right)$.

Let $V_{1}$ be the open set in $X$ defined by

$$
V_{1}=\left\{x \in X\left|e^{+\delta}-\alpha<\right| g(x)\left|<e^{+\delta}+\alpha,\right| h_{v}(x) \mid<1-\varepsilon_{0} \text { for } 1 \leqq v \leqq k\right\} .
$$

Analogously, replacing $e^{+\delta}$ by $e^{-\delta}$ we define an open set $V_{2}$. We set $V=V_{1} \cup V_{2}$. For $x \in V$ and $(\zeta, \eta)$ satisfying $e^{ \pm \rho^{\prime}}-\alpha<|\zeta|<e^{ \pm \rho^{\prime}}+\alpha, 1-\varepsilon^{\prime}-\alpha<\left|\eta_{v}\right| 1-\varepsilon^{\prime}+\alpha$, if $\alpha$ is sufficiently small the function $\chi(x, \zeta, \eta)$ is holomorphic because of assumption (ii). Thus there exist Stein neighborhoods $N_{j}$ of $\Gamma_{j}$ such that $\chi$ is holomorphic in $V \times\left(N_{1} \cup N_{2}\right)$.

Consider the meromorphic function $\chi$ in $X_{3}(\delta) \times N_{j}, 0$ in $T_{j} \times N_{j}$ where $T_{j}$ is the open set $X_{j}(\delta)-\overline{\left(X_{3}(\delta)-V\right)}(j=1,2)$. By the above remark, $\chi$ is holomorphic in $\left(X_{3}(\delta) \cap T_{j}\right) \times N_{j}$. Hence, since $X_{j}(\delta) \times N_{j}$ is a Stein space, there exists a meromorphic function $\chi_{j}$ in $X_{j}(\delta) \times N_{j}$ holomorphic in $T_{j} \times N_{j}$, such that $\chi_{j}-\chi$ is holomorphic in $X_{3}(\delta) \times N_{j}$. Now, by assumption (i), for any $\beta>0$, there is a holomorphic function $\phi_{j}$ on $X_{j}(\delta) \times N_{j}$ such that 


$$
\left|\phi_{j}-\left(\chi-\chi_{j}\right)\right|<\beta \text { on } Y_{3}\left(0, \rho_{0}\right) \times \Gamma_{j} .
$$

Let $\omega_{j}=\chi-\chi_{j}-\phi_{j}$. Then,

$$
\int_{\Gamma_{j}} \chi F d \zeta d \eta=\int_{\Gamma_{j}}\left(\chi_{j}+\phi_{j}\right) F d \zeta d \eta+\int_{\Gamma_{j}} \omega_{j} F d \zeta d \eta
$$

Since $\chi_{j}+\phi_{j}$ is holomorphic on $Y_{j}\left(\varepsilon^{\prime}, \rho^{\prime}\right)$ for any $(\zeta, \eta) \in \Gamma_{j}$, we see that the first integral detines a holomorphic function $f_{j}^{(1)}$ on $Y_{j}\left(\varepsilon^{\prime}, \rho^{\prime}\right)$. Moreover, if $\psi_{j}^{\prime}=\int_{\Gamma_{j}}\left(\omega_{j} F d \zeta d \eta, \quad \psi_{j}^{\prime}\right.$ is holomorphic on $X_{3}(\delta)$ and

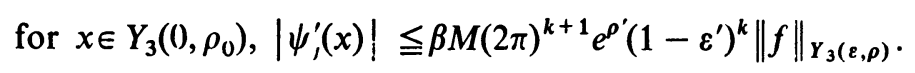

If $\theta^{\prime}=\beta M(2 \pi)^{k+1} e^{\rho^{\prime}}\left(1-\varepsilon^{\prime}\right)^{k}$, we may suppose, by suitable choice of $\beta$, that $\theta^{\prime}<\frac{1}{2}$. Now, we have, for $x \in Y_{3}\left(\varepsilon^{\prime}, \rho^{\prime}\right)$,

$$
f(x)=f_{1}^{(1)}(x)-f_{2}^{(1)}(x)+f^{(1)}(x)
$$

where $f^{(1)}(x)=\psi_{1}^{\prime}(x)-\psi_{2}^{\prime}(x)$. Moreover,

$$
\left\|f^{(1)}\right\|_{Y_{3}\left(0, \rho_{0}\right)} \leqq \theta\|f\|_{Y_{3}(\varepsilon, \rho)}, \quad \theta=2 \theta^{\prime}<1 .
$$

We now proceed with $f^{(1)}$ as we did with $f$ to construct $F^{(1)}$ on $P_{\varepsilon, \rho}$ such that $F^{(1)} \circ \phi=f^{(1)}$ on $Y_{3}(\varepsilon, \rho)$, while $\left\|F^{(1)}\right\|_{P_{\varepsilon^{\prime}, \rho^{\prime}}} \leqq M\left\|f^{(1)}\right\|_{Y_{3}(\varepsilon, \rho)}$. Then, if

$$
f_{j}^{(2)}=\int_{\Gamma_{j}}\left(\chi_{j}+\phi_{j}\right) F^{(1)} d \zeta d \eta
$$

(this being holomorphic on $Y_{j}\left(\varepsilon^{\prime}, \rho^{\prime}\right)$ ), we have

$$
f^{(1)}=f_{1}^{(2)}-f_{2}^{(2)}+f^{(2)}
$$

where $f^{(2)}$ is holomorphic in $X_{3}(\delta)$ and

$$
\left\|f^{(2)}\right\|_{Y_{3}\left(0, \rho_{0}\right)} \leqq \theta\left\|f^{(1)}\right\|_{Y_{3}\left(0, \rho_{0}\right)} \leqq \theta^{2}\|f\|_{Y_{3}(\varepsilon, \rho)} .
$$

Continuing this process, we obtain sequences of holomorphic functions $f^{(p)}$ in $X_{3}(\delta)$ and $F^{(p)}$ in $P_{\varepsilon, \rho}$, such that

$$
\begin{aligned}
& \left\|f^{(p)}\right\|_{Y_{3}\left(0, \rho_{0}\right)} \leqq \theta^{p}\|f\|_{Y_{3}(\varepsilon, \rho)}, \\
& \left\|F^{(p)}\right\|_{P_{\varepsilon^{\prime}, \rho^{\prime}}} \leqq M \theta^{p}\|f\|_{Y_{3}(\varepsilon, \rho)}
\end{aligned}
$$

while if $f_{j}^{(p)}=\int_{\Gamma_{j}}\left(\chi_{j}+\phi_{j}\right) F^{(p)} d \zeta d \eta$, we have, in $Y_{3}\left(\varepsilon^{\prime}, \rho^{\prime}\right)$,

$$
f=\sum_{q=1}^{p}\left(f_{1}^{(q)}-f_{2}^{(q)}\right)+f^{(p)} \text {. }
$$

As $p \rightarrow \infty, f^{(p)} \rightarrow 0$ in $Y_{3}\left(0, \rho_{0}\right)$, and if

$$
f_{j}=\int_{\Gamma_{j}}\left(\chi_{j}+\phi_{j}\right) \sum_{p=1}^{\infty} F^{(p)} d \zeta d \eta,
$$


we have the following properties:

$$
f=f_{1}-f_{2}, \text { in } Y_{3}\left(\varepsilon^{\prime}, \rho^{\prime}\right) .
$$

$f_{j}$ is holomorphic in $Y_{j}\left(\varepsilon^{\prime}, \rho^{\prime}\right)$. For any compact set $K_{j} \subset Y_{j}\left(\varepsilon^{\prime}, \rho^{\prime}\right)$, we have $\left\|f_{j}\right\|_{K_{j}} \leqq \sup _{K_{j}}\left|\chi_{j}+\phi_{j}\right| \cdot(M \theta /(1-\theta))\|f\|_{Y_{3}(\varepsilon, \rho)} \leqq M_{j}\|f\|_{Y_{3}(\varepsilon, \rho)}$ say. This completes the proof of Lemma 2.

(2.3) Third Heftungslemma. Let $X$ be a complex space and $g$ a holomorphic function on $X$ which is nowhere zero. For any $\delta>0$ we define

$$
\begin{aligned}
& X_{1}(\delta)=\left\{x \in X|| g(x) \mid<e^{\delta}\right\}, \\
& X_{2}(\delta)=\left\{x \in X|| g(x) \mid>e^{-\delta}\right\}
\end{aligned}
$$

so that

$$
X=X_{1}(\delta) \cup X_{2}(\delta)
$$

Lemma 3. Suppose that for $\delta=\delta_{0}$ there exists a $C^{\infty}$ strongly pseudoconvex function $p_{i}$ on any relatively compact set $Y_{i} \subset \subset X_{i}\left(\delta_{0}\right), i=1,2$. Then on any relatively compact set $Y \subset \subset X$ there exists $a C^{\infty}$ strongly pseudoconvex function on $Y$.

Proof. Let $U$ be an open subset of $X$ such that

$$
Y \subset \subset U \subset \subset X
$$

Since $U \cap X_{i}\left(c \delta_{0}\right) \subset \subset X_{i}\left(\delta_{0}\right)$ there exists a $C^{\infty}$ strongly pseudoconvex function $p_{i}$ on $U \cap X_{i}\left(c \delta_{0}\right), 1 / 2<c<1$. Consider the function

$$
q_{1}=|g|^{-1}-e^{-\delta_{0} / 2}+\varepsilon p_{1}
$$

on the set $U \cap X_{1}\left(c \delta_{0}\right)$. Since $g^{-1}$ is holomorphic on $X, q_{1}$ is a $C^{\infty}$ strongly pseudoconvex function for every $\varepsilon>0$. If $\varepsilon$ is sufficiently small, the set

$$
W_{1}=\left\{x \in U \cap X_{1}\left(c \delta_{0}\right) \mid q_{1}(x)>0\right\}
$$

contains the set $Y \cap X_{1}\left(\delta_{0} / 3\right)$, since on this set

$$
|g|^{-1}-e^{-\delta_{0} / 2}>e^{-\delta_{0} / 3}-e^{-\delta_{0} / 2} \text { and } Y \cap X_{1}\left(\delta_{0} / 3\right) \subset \subset U \cap X_{1}\left(\delta_{0} / 2\right)
$$

Moreover on the boundary of $W_{1} \cap Y$ in $Y, q_{1}$ is defined and equal to zero, since, if $\varepsilon$ is sufficiently small, $q_{1}$ is negative on the set $\left\{x \in X|| g \mid=e^{(c-8) \delta_{0}}\right\} \cap U$.

Consider for any constant $\lambda>0$ the function $\phi_{1}$ on $Y$ defined by

$$
\phi_{1}(x)= \begin{cases}e^{-\lambda / q_{1}(x)} & \text { for } x \in W_{1} \cap Y, \\ 0 & \text { for } x \in Y-\left(W_{1} \cap Y\right) .\end{cases}
$$

This is a $C^{\infty}$ function on $Y$ which is pseudoconvex and also strongly pseudoconvex on $Y \cap X_{1}\left(\delta_{0} / 3\right)$, if $\lambda>2 \sup \left\{c_{1}(x), x \in Y \cap X_{1}\left(\delta_{0} / 3\right)\right\}$. 
Using the functions $g, p_{2}$ instead of $g^{-1}, p_{1}$ we construct similarly a $C^{\infty}$ function $\phi_{2}(x)$ on $Y$ which is pseudoconvex and also strongly pseudoconvex on $Y \cap X_{2}\left(\delta_{0} / 3\right)$. Then the function $\phi_{1}+\phi_{2}$ is $C^{\infty}$ and strongly pseudoconvex on $Y$.

3. Levi problem. The object of this section is a proof of the following theorem [9].

THEOREM 1. Let $X$ be a complex space and $p$ a continuous strongly pseudoconvex function on $X$ such that for any $\alpha>0$ the set

$$
X_{\alpha}=\{x \in X \mid p(x)<\alpha\}
$$

is relatively compact in $X$. Then $X$ is a Stein space.

Because of [9, Lemma 5], we may suppose that $p$ is locally the maximum of finitely many $C^{\infty}$ strongly pseudoconvex functions. We assume this in what follows. The proof then consists of two parts.

(3.1) If $X_{\alpha}$ is a Stein space for some $\alpha$, then there is $\alpha^{\prime}>\alpha$ such that $X_{\alpha^{\prime}}$ is a Stein space.

(3.2) If $\alpha_{v}<\alpha, \alpha_{v} \rightarrow \alpha$ as $v \rightarrow \infty$ and $X_{\alpha_{v}}$ is a Stein space for each $v$, then $X_{\alpha}$ is also a Stein space.

For the proof of (3.1) we need the following lemma.

LEMMA 4. Let $X$ be a complex space and $p$ a function with the properties given above. Suppose in addition there is a continuous complex-valued function $f$ on $X$ such that for some $\rho>0$ the sets

$$
X_{1}(\rho)=\{x \in X \mid \operatorname{Re} f<+\rho\}, \quad X_{2}(\rho)=\{x \in X \mid \operatorname{Re} f>-\rho\}
$$

are Stein spaces, while $f$ is holomorphic in $X_{3}(\rho)=X_{1}(\rho) \cap X_{2}(\rho)$. Then $X$ itself is a Stein space.

Proof. For any $\alpha>0$, consider the set

$$
\{x \in X \mid p(x)<\alpha\}=X_{\alpha} .
$$

It follows from (1.5) that there exists $\beta>\alpha$ such that for any $x_{0} \in \partial X_{\alpha}$ there is an analytic set $A_{x_{0}}$ in $X_{\beta}$ and a holomorphic function $f_{x_{0}}$ in a neighborhood $U_{x_{0}}$ of $A_{x_{0}}$ in $X_{\beta}$ satisfying the conditions

$$
A_{x_{0}} \cap \bar{X}_{\alpha}=\left\{x_{0}\right\}, \quad A_{x_{0}}=\left\{x \in U_{x_{0}} \mid f_{x_{0}}(x)=0\right\} .
$$

For $0<\delta \leqq \rho$, let $\Omega_{j}(\delta)=X_{j}(\delta) \cap X_{\beta}$, and let $\Omega_{j}^{\alpha}=\Omega_{j}(\rho) \cap X_{\alpha}$. Since $\Omega_{3}(\rho)$ is Stein, there is, for $x_{0}$ in $\Omega_{3}(\mathrm{c} \rho) \cap \partial X_{\alpha}, 1 / 3<c<1 / 2$, a meromorphic function $\psi_{x_{0}}$ in $\Omega_{3}(\rho)$, holomorphic in $\Omega_{3}(\rho)-A_{x_{0}}$, for which $\psi_{x_{0}}-1 / f_{x_{0}}$ is holomorphic in $U_{x_{0}} \cap \Omega_{3}(\rho)$. Now, $\psi_{x_{0}}$ is bounded on the set $\Omega_{3}^{\alpha} \cap\left\{x \in X_{3}(\rho)|| e^{f} \mid=e^{ \pm \rho / 2}\right\}$ and on any compact subset of $\Omega_{3}^{\alpha}$, while $\left|\psi_{x_{0}}(x)\right| \rightarrow \infty$ as $x \rightarrow x_{0}, x \in X_{\alpha^{*}}$. It fol- 
lows that given any compact subset $K$ of $X_{\alpha}$, we can construct finitely many holomorphic functions $h_{1}, \cdots, h_{k}$ on $\Omega_{3}^{\alpha}$ having the following properties. Put $g=e^{f}$ in $X_{3}(\rho)$. Then

(a) There is an $\varepsilon_{0}>0$ such that

$$
\left|h_{v}(x)\right|<1-\varepsilon_{0} \text { for } x \in \Omega_{3}^{\alpha} \cap\left\{y \in X_{3}(\rho)|| g(y) \mid=e^{ \pm \rho / 2}\right\} .
$$

(b) If $0 \leqq \varepsilon<\varepsilon_{0}$, the set

$$
Y(\varepsilon)=\left(X_{\alpha}-\Omega_{3}^{\alpha}\right) \cup\left\{x \in \Omega_{3}^{\alpha}|| h_{\nu}(x) \mid<1-\varepsilon, v=1, \cdots, k\right\}
$$

contains $K$.

(c) The set $\Omega_{3}(\rho / 3) \cap Y(0)$ is relatively compact in $X$ and the functions $h_{1}, \cdots, h_{k}$ separate points and give local coordinates on it.

We can apply now the second Heftungslemma to the space $X_{\alpha}$ since by (1.2) and (1.4), the open sets $\Omega_{1}^{\alpha}, \Omega_{2}^{\alpha}$ are Stein and $\Omega_{3}^{\alpha}$ is Runge in each of them. Hence,

(*) for any compact sets $C_{j} \subset \Omega_{j}^{\alpha}$, there exists a compact subset $C$ of $\Omega_{3}^{\alpha}$ such that the following result holds: there exist constants $M>0$ and $\delta>0$ such that for any holomorphic function $h$ in $\Omega_{3}^{\alpha}$, there exist holomorphic functions $h_{j}(j=1,2)$ in $Y(\varepsilon) \cap \Omega_{j}(\delta)$, such that $h_{1}-h_{2}=h$ on $Y(\varepsilon) \cap \Omega_{3}(\delta)$, while $\left\|h_{j}\right\|_{c_{j}} \leqq M\|h\|_{c}$.

We assert now that for any $\gamma>0$, the set $X_{\gamma}$ is Stein. To prove this, let $\alpha>\gamma$ be so chosen that for any $x_{0} \in \partial X_{\gamma}$, there exists an analytic set $B_{x_{0}}$ in $X_{\alpha}$, a neighborhood $U_{x_{0}}$ of $B_{x_{0}}$ in $X_{\alpha}$ and a holomorphic function $g_{x_{0}}$ in $U_{x_{0}}$ such that $B_{x_{0}} \cap \bar{X}_{\gamma}=\left\{x_{0}\right\}$ and $B_{x_{0}}=\left\{x \in U_{x_{0}} \mid g_{x_{0}}(x)=0\right\}$. Let $\psi_{j}$ be meromorphic in $\Omega_{j}^{\alpha}$ and such that $\psi_{j}$ is holomorphic in $\Omega_{j}^{\alpha}-B_{x_{0}}, \psi_{j}-1 / g_{x_{0}}$ is holomorphic in $U_{x_{0}} \cap \Omega_{j}^{\alpha}$; finally let $h=\psi_{1}-\psi_{2}$ in $\Omega_{3}^{\alpha}$. Clearly $h$ is holomorphic in $\Omega_{3}^{\alpha}$.

We now place ourselves in the situation of the Heftungslemma in such a way that $Y(\varepsilon) \supset \bar{X}_{\gamma}$ and find functions $h_{j}$ in $\Omega_{j}(\delta) \cap Y(\varepsilon)$ with $h_{1}-h_{2}=h$. Then the function $\psi$ in $Y(\varepsilon)$ defined to be $\psi_{j}-h_{j}$ in $Y(\varepsilon) \cap \Omega_{j}(\delta)$ is meromorphic in $Y(\varepsilon)$, holomorphic in $Y(\varepsilon)-B_{x_{0}}$, and $\psi-1 / g_{x_{0}}$ is holomorphic in $U_{x_{n}} \cap Y(\varepsilon)$. Clearly the restriction of $\psi$ to $X_{\gamma}$ is a holomorphic function and $|\psi(x)| \rightarrow \infty$ as $x \rightarrow x_{0}$. Since $x_{0} \in \partial X_{\gamma}$ is arbitrary, $X_{\gamma}$ is holomorph-convex.

Let $x_{1} \neq x_{2}$ be two points of $X_{\gamma}$ and suppose first that both are points of $\Omega_{1}^{\alpha}$. Let $\phi$ be holomorphic in $\Omega_{1}^{\alpha}$ and $\phi\left(x_{1}\right) \neq \phi\left(x_{2}\right)$. Take $C_{1}=\left\{x_{1}\right\} \cup\left\{x_{2}\right\}$ and let $C \subset \Omega_{3}^{\alpha}$ be as in $\left(^{*}\right)$ above. Let $\phi^{\prime}$ be holomorphic in $\Omega_{2}^{\alpha}$ and, with $h=\phi-\phi^{\prime}$, let $\|h\|_{c}<\varepsilon$ (such a $\phi^{\prime}$ exists since $\Omega_{3}^{\alpha}$ is Runge in $\Omega_{2}^{\alpha}$ ). As above, there are holomorphic functions $h_{j}$ on $Y(\varepsilon) \cap \Omega_{j}^{\alpha}$ with $\left\|h_{1}\right\|_{c_{1}}<M \varepsilon, h_{1}-h_{2}=h$ in $Y(\varepsilon) \cap \Omega_{3}(\delta)$. The function $\psi$ defined to be $\phi-h_{1}$ in $Y(\varepsilon) \cap \Omega_{1}(\delta), \phi^{\prime}-h_{2}$ in $Y(\varepsilon) \cap \Omega_{2}(\delta)$ is holomorphic in $Y(\varepsilon)\left(\supset X_{\gamma}\right)$ and $\|\psi-\phi\|_{c_{1}}<M \varepsilon$. Since $\varepsilon>0$ is arbitrary, we can choose it such that $\psi\left(x_{1}\right) \neq \psi\left(x_{2}\right)$. In the case $x_{1} \in \Omega_{1}^{\alpha}$, $x_{2} \in \Omega_{2}^{\alpha}$, we take $C_{1}=\left\{x_{1}\right\}, C_{2}=\left\{x_{2}\right\}$ and the compact set $C \subset \Omega_{3}^{\alpha}$ as in (*) above. We then find a holomorphic function $\phi_{j}$ in $\Omega_{j}^{\alpha}$ such that $\left|\phi_{1}\left(x_{1}\right)-\phi_{2}\left(x_{2}\right)\right|$ 
$\geqq 1$ and such that $\left\|\phi_{1}-\phi_{2}\right\|_{c}<\varepsilon$. Then, as above, we can find a holomorphic function $\psi$ on $Y(\varepsilon)$ such that $\left\|\psi\left(x_{j}\right)-\psi_{j}\left(x_{j}\right)\right\|<M \varepsilon$. Clearly if $\varepsilon<1 / M, \psi\left(x_{1}\right) \neq \psi\left(x_{2}\right)$. A similar argument applies when $x_{1}, x_{2} \in \Omega_{2}^{\alpha}$. Thus we conclude that holomorphic functions separate points of $X_{\gamma}$.

The proof that holomorphic functions on $X_{\gamma}$ give local coordinates at each point is similar and so is omitted.

This completes the proof that $X_{\gamma}$ is a Stein space for any $\gamma>0$.

By (1.1) and (1.2), we conclude that $X$ is a Stein space, which proves Lemma 4.

We now go to the proof of (3.1).

Let $\alpha$ be such that $X_{\alpha}$ is a Stein space. Now, by (1.5), there is $\beta>\alpha$ such that if $p\left(x_{0}\right)=\alpha$, there exists a Stein neighborhood $U_{x_{0}}$ and a holomorphic function $\phi$ in $U_{x_{0}}$ such that

(i) $B=\left\{x \in U_{x_{0}} \mid \operatorname{Re} \phi(x)=0\right\}$ is closed in $X_{\beta}$;

(ii) $B \cap Y_{\alpha}=\left\{x_{0}\right\}$ where $Y_{\alpha}=\{x \in X \mid p(x) \leqq \alpha\}$.

We may further suppose that if $x \in U_{x_{0}} \cap Y_{\alpha}$, then $\operatorname{Re} \phi(x) \leqq 0$. Let now $\beta^{\prime}, \alpha<\beta^{\prime}<\beta$ be fixed.

If $c<0$ is sufficiently near to zero, then we have

(a) $B_{c}=\left\{x \in U_{x_{0}} \mid \operatorname{Re} \phi(x)=c\right\}$ is closed in $X_{\beta^{\prime}}$;

(b) $V_{c}=\left\{x \in U_{x_{0}} \mid \operatorname{Re} \phi(x)>c\right\}$ is a neighborhood of $x_{0}$ such that $\operatorname{Re} \phi(x)=c$ if $x \in \partial V_{c} \cap X_{\beta^{\prime}}$.

Let $c<d<0$; the set $V_{d}$ is also a neighborhood of $x_{0}$.

We now find points $x_{0}, \cdots, x_{p} \in\{x \in X \mid p(x)=\alpha\}$ and corresponding sets $V_{c_{v}}, V_{d_{v}}$ as above (the corresponding functions being $\phi_{v}$ ) such that the $V_{d_{v}}$ form a covering of $\{x \in X \mid p(x)=\alpha\}$. We find $C^{\infty}$ function $l_{v}$ with compact support contained in $V_{d_{v}} \cap X_{\beta^{\prime}}$, such that $p \pm l$ is strongly convex in $X_{\beta^{\prime}}, l$ being any finite sum of the $l_{v}$, while $\sum_{v=0}^{p} l_{v}(x)>0$ for any $x$ with $p(x)=\alpha$.

Consider the space

$$
D_{q}=\left\{x \in X \mid p(x)<\alpha+l_{0}(x)+\cdots+l_{q}(x)\right\},
$$

$q=-1, \cdots, p$. Suppose that $D_{q}$ is a Stein space. We assert that then $D_{q+1}$ is also a Stein space. To prove this we proceed as follows. We define the function $f_{q+1}$ in $D_{q+1}$ in the following way: $\operatorname{Re} f_{q+1}(x)=c_{q+1}$ for $x$ in $D_{q+1}-U_{x_{n+1}}$, $\operatorname{Re} f_{q+1}(x)=\max \left(c_{q+1}, \operatorname{Re} \phi_{q+1}(x)\right)$ for $x \in U_{x_{q+1}}$, while $\operatorname{Im} f_{q+1}$ is any continuous extension to $D_{q+1}$ of the restriction of $\operatorname{Im} \phi_{q+1}$ to $D_{q+1} \cap V_{d_{n+1}}$. Then $f_{q+1}$ is holomorphic on the set $\left\{x \in D_{q+1} \mid c_{q+1}<\operatorname{Re} f_{q+1}<d_{q+1}\right\}$. Now, since $D_{q}$ is a Stein space the set $\left\{x \in D_{q+1} \mid \operatorname{Re} f_{q+1}<d_{q+1}\right\}=\left\{x \in D_{q} \mid \operatorname{Re} f_{q+1}<d_{q+1}\right\}$ is a Stein space (by (1.4)) while, since $U_{x_{q+1}}$ is a Stein space, the set $\left\{x \in D_{q+1} \mid \operatorname{Re} f_{q+1}>c_{q+1}\right\}=\left\{x \in U_{x_{n+1}} \mid \operatorname{Re} f_{q+1}>c_{q+1}\right\}$ is a Stein space (again by (1.4)). Moreover, in $D_{q+1}$, there is a strongly pseudoconvex function $P$ with $\left\{x \in D_{q+1} \mid P(x)<r\right\} \subset \subset D_{q+1}$ for any $r>0$ (in fact, we may take $\left.P(x)=\left(\alpha-p(x)+l_{0}(x)+\cdots+l_{q+1}(x)\right)^{-1}\right)$. Hence, by Lemma $4, D_{n+1}$ is Stein. 
Since by supposition, $D_{-1}=X_{\alpha}$ is Stein, it follows that $D_{p}$ is Stein. Since $D_{p}$ is a neighborhood of $Y_{\alpha}$, we may choose $\alpha^{\prime}>\alpha$ so that $X_{\alpha^{\prime}} \subset D_{p}$. Then, by (1.2), $X_{\alpha^{\prime}}$, is Stein. This completes the proof of (3.1).

Proof of (3.2). This is a simple consequence of the Runge theorem (1.2) and (1.1).

Proof of Theorem 1. Let $E$ be the set of real $\alpha$ for which $X_{\alpha}$ is Stein ( $E$ is nonempty since it contains all $\alpha$ for which $\left.X_{\alpha}=\varnothing\right)$. Clearly, if $\alpha \in E$ and $\gamma<\alpha$, then $\gamma \in E$. By (3.1), $E$ is open. By (3.2), $E$ is closed. Hence $E$ is the whole real line. Hence, for any $\alpha, X_{\alpha}$ is Stein. Again by the Runge theorem, $X$ itself is Stein.

4. Pseudoconvex domains in $K$-complete spaces. Let $D$ be a relatively compact open set on the complex space $X . D$ is called pseudoconvex if to any $x_{0} \in \partial D$, there is a neighborhood $U_{x_{0}}$ in $X$ and a continuous pseudoconvex function $p_{x_{0}}$ in $U_{x_{0}}$ with

$$
D \cap U_{x_{0}}=\left\{x \in U_{x_{0}} \mid p_{x_{0}}(x)<0\right\} .
$$

It is called a pseudoconvex domain with globally defined boundary if there is a neighborhood $U$ of $\partial D$ and a continuous pseudoconvex function $p$ in $U$ such that $D \cap U=\{x \in U \mid p(x)<0\}$. The object of this section is to prove the following

THEOREM 2. A relatively compact open set $D$ on the $K$-complete complex space $X$ which is pseudoconvex with globally defined boundary is a Stein space.

Before giving the proof of Theorem 2, we prove the following lemma, which is of independent interest.

Lemma 5. Let $X$ be a K-complete space and $D \subset \subset X$ an open subset. Then, there exists a $C^{\alpha_{i}}$ strongly pseudoconvex function in $D$.

The proof is based on

Lemma 6. Let $X, Y$ be two locally compact spaces and $\phi: X \rightarrow Y$ a continuous map with discrete fibres. Let $K$ be a compact set in $X$ and $W_{1}, \cdots, W_{k}$ a finite open covering of $K$ in $X$. Then there exists a finite open covering $V_{1}, \cdots, V_{p}$ of $\phi(K)$ in $Y$ such that every connected component of $\phi^{-1}\left(V_{v}\right)$ which meets $K$ is contained in some $W_{\mu}$.

Proof. It is enough to prove that for any $y \in \phi(K)$, there is an open neighborhood $V_{y}$ of $y$ such that any connected component of $\phi^{-1}\left(V_{y}\right)$ which meets $K$ is contained in some $W_{\mu}$. Let $x_{1}, \cdots, x_{q}$ be the points of $K$ with $\phi\left(x_{j}\right)=y$ and let $K^{\prime}$ be a compact neighborhood of $K$ containing no other point of $\phi^{-1}(y)$. Let $U_{j}$ be an open neighborhood of $x_{j}$ with $U_{j} \subset W_{\mu} \cap K^{\prime}$ for some $\mu, U_{j} \cap U_{j^{\prime}}=\varnothing$ if $j \neq j^{\prime}$. Now $C=\phi\left(\partial K^{\prime}\right) \cup \phi\left(K^{\prime}-\bigcup_{j \leq q} U_{j}\right)$ is a compact subset of $Y$ not containing $y$. Let $V_{y}$ be an open neighborhood of $y$ not meeting $C$. Then 
$\phi^{-1}\left(V_{y}\right) \cap K^{\prime} \subset \bigcup_{j \leqq q} U_{j}$, and $\phi^{-1}\left(V_{y}\right) \cap \partial K^{\prime}=\varnothing$. Hence any connected component of $\phi^{-1}\left(V_{y}\right)$ meeting $K$ (or $K^{\prime}$ ) is contained in $\bigcup U_{j}$, and since the $U_{j}$ are disjoint, in one of them. Since each $U_{j}$ is contained in some $W_{\mu}$, Lemma 6 is proved.

Proof of Lemma 5. We may suppose that there is a holomorphic map $\phi: X \rightarrow C^{N}$ with discrete fibres. Let $\left\{W_{1}, \cdots, W_{k}\right\}$ be a finite covering of $\bar{D}$ by open sets in each of which there exists a $C^{\infty}$ strongly convex function. Let $V_{1}, \cdots, V_{p}$ be an open covering of $\phi(\bar{D})$ in $C^{N}$ such that each connected component of $\phi^{-1}\left(V_{v}\right) \subset W_{\mu}$ for some $\mu$. We divide $C^{N}$ into closed cubes of side $\varepsilon>0$ : if $z_{j}=x_{j}+i x_{N+j}$, consider the cube (1) $K_{n, \varepsilon}=\left\{\varepsilon n_{j} \leqq x_{j} \leqq \varepsilon\left(n_{j}+1\right)\right\}$, $n=\left(n_{1}, \cdots, n_{2 N}\right), n_{j}$ integers. For sufficiently small $\varepsilon$, if $K_{n, \varepsilon} \cap \phi(\bar{D}) \neq \varnothing$, then $K_{n, \varepsilon} \subset V_{r}$ for some $r$.

Let $U$ be a neighborhood of $\bar{D}$ contained in $\bigcup W_{\mu}$. Let $K_{n}$, be the union of the $K_{n, \varepsilon}$ with fixed $n^{\prime}=\left(n_{2}, \cdots, n_{2 N}\right)$, which meet $\phi(\bar{D})$. Then, by successive applications of the Heftungslemma Lemma 3, we see that there is a neighborhood $V_{n^{\prime}}$ of $K_{n}$, such that any relatively compact subset of $\phi^{-1}\left(V_{n^{\prime}}\right) \cap U$ carries a $C^{\infty}$ strongly convex function. If $K_{n^{\prime \prime}}$ is the union of the sets $K_{n^{\prime}}$ with fixed $n^{\prime \prime}=\left(n_{3}, \cdots, n_{2 N}\right)$, we see, in the same way that there is a neighborhood $V_{n^{\prime \prime}}$ of $K_{n^{\prime \prime}}$ such that any relatively compact subset of $\phi^{-1}\left(V_{n^{\prime \prime}}\right) \cap U$ carries a $C^{\infty}$ strongly convex function. Repeating this process, there is a neighborhood $V$ of $\phi(\bar{D})$ for which any relatively compact subset of $\phi^{-1}(V) \cap U$ (in particular $D$ ) carries a $C^{\infty}$ strongly pseudoconvex function, which gives Lemma 5.

It is now simple to give the proof of Theorem 2.

Proof of Theorem 2. Let $X$ be $K$ complete, $D \subset \subset X$ and $U$ a neighborhood of $\partial D$ in which there exists a continuous pseudoconvex function $p$ with $U \cap D=\{x \in U \mid p(x)<0\}$. We assert that there is a neighborhood $V$ of $\bar{D}$ and a continuous convex function $p^{\prime}$ in $V$ with $D=\left\{x \in V \mid p^{\prime}(x)<0\right\}$. In fact let $D^{\prime} \subset \subset D$ and $\partial D^{\prime} \subset U$; let $-\delta=\operatorname{Sup} p(x)$ for $x \in \partial D^{\prime} \cap U$. Then clearly $-\delta<0$. Let $p^{\prime}(x)=-\delta$ in $D^{\prime}, p^{\prime}(x)=\max (p(x),-\delta)$ for $x \in U-D^{\prime}$. We may take $V=D \cup U$. Because of Lemma 5 , there is a strongly pseudoconvex function $q$ in D. Let

$$
P(x)=-\frac{1}{p^{\prime}(x)}+e^{q(x)} \text { for } x \in D .
$$

Then $P(x)$ is strongly convex and for any $r$, the set $\{x \in D \mid P(x)<r\} \subset \subset D$. By Theorem $1, D$ is Stein.

From Theorem 2 and the Runge theorem (1.2) we deduce

THEOREM 3. Let $X$ be a K-complete complex space. If there exists a continuous pseudoconvex function $p$ on $X$ such that for any $\alpha>0$ the set $\{x \in X \mid p(x)<\alpha\}$ is relatively compact, then $X$ is Stein.

5. Pseudoconvex domains in Stein spaces. In Theorem 1, we had to sup- 
pose that the boundary of the domain $D$ in $X$ was globally defined. For domains spread over $C^{n}$, the method of Oka provides a method to reduce the case of arbitrary pseudoconvex domains to this. Since we shall require his result for our next theorem, we state it as a separate lemma. The proof is contained in [11]; see also [3].

Lemma 7. Let $X$ be a complex space of pure dimension $n$ and $\phi: X \rightarrow C^{n} a$ holomorphic map. Let $U$ be the set of points of $X$ where $\phi$ is a local isomorphism, and let $D \subset \subset X$, be an open set contained in $U$. Suppose that every point of $\partial D$ has a neighborhood $W$ in $X$ such that $W \cap D$ is Stein. Then, if for $x \in D, d(x)$ denotes the "radius" of the largest connected open subset of $D$ which is isomorphic, by $\phi$, to a polycylinder about $\phi(x)$, then $-\log d(x)$ is a pseudoconvex function in $D$.

(Here "polycylinder" means a set of the form $\left|z_{i}-z_{i}^{(0)}\right|<r, r$ not depending on i.)

THEOREM 4. Let $X$ be a Stein space, $S$ the set of its singular points. Let $D$ be an open subset of $X$ such that every point of $\partial D$ has a neighborhood $W$ in $X$ for which $W \cap D$ is Stein. Moreover, suppose that there is a neighborhood $U$ of $S \cap \partial D$ such that $D \cap U$ is a Stein space.

Then $D$ itself is a Stein space.

Proof. We will first prove the theorem under the additional assumption that $X$ is irreducible; suppose that $\operatorname{dim}_{c} X=n$.

We may suppose $D$ to be relatively compact in $X$. In fact if $\Delta$ is any Runge domain in $X, \Delta \subset \subset X$, it is enough to prove that $D^{\prime}=\Delta \cap D$ is Stein. But $D^{\prime}$ satisfies the same conditions as $D$.

We assert that for any $U^{\prime}$ with $D \subset U^{\prime} \subset \subset X$, there are finitely many holomorphic maps

$$
f^{(v)}: X \rightarrow C^{n}, \quad v=1, \cdots, k,
$$

and holomorphic functions $g_{v}$ on $X$ such that if we set $A_{v}=\left\{x \in X \mid g_{v}(x)=0\right\}$, the following conditions are fulfilled: (i) each $f^{(v)}$ has discrete fibres in $X$, (ii) $f^{(v)}: U^{\prime}-A_{v} \rightarrow C^{n}$ is a local isomorphism and (iii) $\bigcap_{v=1}^{k} A_{v} \cap U^{\prime}=S \cap U^{\prime}$.

Since each analytic set in $X$ is the set of common zeros of finitely many holomorphic functions, it is sufficient to find holomorphic mappings $f^{(v)}$ and analytic sets $A_{v}$ satisfying (i), (ii), (iii).

To do this, we note first the following:

Given finitely many points $x_{1}, \cdots, x_{p} \in X-S$, there is a holomorphic map $f: X \rightarrow C^{n}$ with discrete fibres in $X$ such that the jacobian determinant $j_{f}$ of $f$ at $x_{v}$ is $\neq 0, v=1, \cdots, p$. This is an immediate consequence of the fact that the holomorphic maps of $X$ into $C^{n}$ with discrete fibres in $X$ are dense in the space of all holomorphic maps of $X$ into $C^{n}$ 
To complete the construction of $f^{(v)}, A_{v}$, suppose that these are already constructed for $v \leqq l-1$ and choose one point $x_{r}, r=1, \cdots, p$, from each irreducible component of $A_{1} \cap \cdots \cap A_{l-1}$ which meets $U^{\prime}-S$; and construct $f^{(l)}: X \rightarrow C^{n}$ so that it has discrete fibres, while $j_{f(l)}\left(x_{r}\right) \neq 0$; and define $A_{l}$ to be the closure in $X$ of the set of points $x \in X-S$ where $j_{f^{(1)}}(x)=0 . A_{l}$ is an analytic set by [10, Satz 16], and since

$$
\operatorname{dim}\left(A_{1} \cap \cdots \cap A_{l-1} \cap\left(U^{\prime}-S\right)\right) \leqq \operatorname{dim}\left(A_{1} \cap \cdots \cap A_{l} \cap\left(U^{\prime}-S\right)\right)-1,
$$

the mappings $f^{(v)}, v \leqq n+1$, satisfy our requirements.

We define $U_{v}=\left\{x \in U^{\prime} \mid g_{v}(x) \neq 0\right\}, D_{v}=D \cap U_{v}$. Now, the pair $\left(U_{v}, f^{(v)}\right)$ is an unramified domain over $C^{n}$, and we denote by $d_{v}(x)$ the polycylindrical distance of $x$ from the boundary of $U_{v}$ (see statement of Lemma 7). Let $\delta_{v}(x)$, $x \in D_{v}$, denote the distance of $x$ from the boundary of $D_{v}$. Then we have

(a) $\delta_{v}(x) \leqq d_{v}(x)$ for $x \in D_{v}$,

(b) $-\log \delta_{v}(x)$ is pseudoconvex in $D_{v}$ (Lemma 7 ).

We now assert that the following two results hold.

(5.1) There is a neighborhood $V_{v}$ of $A_{v} \cap D$ such that for $x \in V_{v} \cap D_{v}$, we have

$$
\delta_{v}(x)=d_{v}(x)
$$

(5.2) There is an integer $h>0$ and a constant $C>0$ such that for any $x \in D_{v}$, we have

$$
\left|g_{v}(x)\right| \leqq C\left\{d_{v}(x)\right\}^{1 / h}
$$

Proof of (5.1). We have only to show that for any $x_{0} \in A_{v} \cap D$, there is a neighborhood $W$ such that for $x \in W-A_{v}$, we have $\delta_{v}(x)=d_{v}(x)$. Now choose a connected neighborhood $W_{1} \subset D$ which is a ramified covering, by means of $f^{(v)}$, of a polycylinder of radius $r$ about $z_{0}=f^{(v)}\left(x_{0}\right)$ and which contains no other point $x_{1}$ with $f^{(v)}\left(x_{1}\right)=z_{0}$. Let $W$ be the inverse image in $W_{1}$ of the polycylinder of radius $\frac{1}{2} r$ about $z_{0}$. If $x \in W-A_{v}$, the "maximal polycylinder about $x$ in $U_{v}$ ", cannot contain $x_{0}$, so has radius $<\frac{1}{2} r$ and so is contained in $W_{1} \subset D$. Hence, for $x \in W-A_{v}$, we have $d_{v}(x)=\delta_{v}(x)$.

Proof of (5.2). For $x_{0} \in A_{v} \cap \bar{D}$, let $W=W\left(x_{0}\right)$ be a connected, relatively compact open set, containing no other point of $x$, with $f^{(v)}\left(x_{1}\right)=f^{(v)}\left(x_{0}\right)$ and such that $f^{(v)} \mid W$ is a ramified covering of a polycylinder $Z$ of radius $r$ in $C^{n}$ about $z_{0}=f^{(v)}\left(x_{0}\right)$. For the sake of simplicity, we drop the indices $v$ in the rest of the proof of (5.2). We denote by $Z_{1}$ the polycylinder of radius $\frac{2}{3} r$ about $z_{0}$.

Let $W^{\prime}$ be the inverse image in $W$ of the polycylinder $Z^{\prime}$ about $z_{0}$ of radius $\frac{1}{3} r$, and let $S(x)$, for $x \in W^{\prime}-A$, be the maximal polycylinder (of radius $d(x)$ ) about $x$ in $X-A$. Then $\partial S(x) \cap A \neq \varnothing$, and $f(S(x))$ is contained in the polycylinder $Z_{1}$.

For $x \in W$ and $z=f(x), w=g(x)\left(=g_{v}(x)\right)$ satisfies an equation

$$
w^{p}+a_{1}(z) w^{p-1}+\cdots+a_{p}(z)=0,
$$


where the $a_{i}$ are bounded holomorphic functions on $Z$. Moreover for $z^{\prime}, z^{\prime \prime} \in Z_{1}$, we have an inequality

$$
\left|a_{i}\left(z^{\prime}\right)-a_{i}\left(z^{\prime \prime}\right)\right| \leqq C_{1}\left|z^{\prime}-z^{\prime \prime}\right| .
$$

Suppose now that $x \in W^{\prime}-A$ and $\xi \in S(x), f(x)=z, f(\xi)=\zeta$. Then $z, \zeta \in Z_{1}$, and from the equation (1) satisfied by $g$ in $S(x)$ it follows that $\left({ }^{2}\right)$

$$
\begin{aligned}
|g(x)-g(\xi)| & \leqq C_{2} \sup _{0 \leqq 1, i}\left|a_{i}(z)-a_{i}(z z+(1-t) \zeta)\right|^{1 / p} \\
& \leqq C_{3}|z-\zeta|^{1 / p} \leqq C_{4}[d(x)]^{1 / p} .
\end{aligned}
$$

If we let $\xi$ tend to a point of $\partial S(x) \cap A$, then $g(\xi)$ tends to zero and we obtain, for $x \in W^{\prime}-A$,

$$
|g(x)| \leqq C_{4}[d(x)]^{1 / p} .
$$

The proof of (5.2) follows immediately from this.

Consider on $D_{v}$ the function

$$
p_{v}^{\prime}(x)=-\log \delta_{v}(x)+h \log \left|g_{v}(x)\right|-\log C,
$$

where $C, h$ satisfy (5.2). Now, because of remark (b) before (5.1) $p_{v}^{\prime}(x)$ is pseudoconvex in $D_{v}$. By (5.1) and (5.2), there is a neighborhood $V_{v}$ of $A_{v} \cap D$ such that if $x \in V_{v} \cap D_{v}$, we have $p_{v}^{\prime}(x) \leqq 0$. Hence the function

$$
p_{v}(x)= \begin{cases}\max \left(p_{v}^{\prime}(x), 0\right) & \text { for } x \in D_{v}, \\ 0 & \text { for } x \in V_{v}\end{cases}
$$

is a pseudoconvex function in $D$, and if $x_{0} \in \partial D-A_{v}$, then $p_{v}(x) \rightarrow \infty$ as $x \in D, x \rightarrow x_{0}$. Hence, if $p(x)=\max p_{v}(x)$, then $p$ is pseudoconvex in $D$ and $p(x) \rightarrow \infty$ if $x \rightarrow x_{0} \notin \bigcap A_{v} \cap \partial D=S \cap \partial D$. Also, if $x \in S \cap D$, we have $p(x)=0$. To complete the proof of Theorem 4 in the special case, we have only to show that for any $\alpha>0$, the set

$$
D_{\alpha}=\{x \in D \mid p(x)<\alpha\}
$$

is a Stein space.

Suppose that $U$ is a neighborhood of $\partial D \cap S$ such that $V=U \cap D$ is Stein. Let $s(x)$ be a pseudoconvex function in $V$ such that for any $r>0$, the set $\{x \in V \mid s(x)<r\} \subset \subset V$. Let $\Omega \subset \subset U$ be a neighborbood of $\partial D \cap S$. Now $\bar{D}_{x} \cap \partial D \subset \partial D \cap S$ since $p(x) \rightarrow \infty$ if $x \rightarrow x_{0} \in \partial D, x_{0} \notin S$. Hence $s(x)$ is bounded for $x \in \partial \Omega \cap D_{\alpha}$, say $s(x) \leqq M$. Consider the function $s^{\prime}$ on $D_{\alpha}$ defined to be

( $\left.{ }^{2}\right)$ We have, in fact the following lemma (see, e.g., Lokasiewicz [7]). Let $B$ be a connected open set in $R^{m}$ and $f$ a continuous function on $B$ satisfying $f^{p}(x)+\sum_{1 \leqq i \leqq p} a_{i}(x) f^{p-i}(x)=0$ where the $a_{i}(x)$ are bounded continuous functions on $B,\left|a_{i}(x)\right| \leqq T$. Then, there is a constant $M=M(T)$ such that if $x, x^{\prime} \in B$ and $\gamma$ is a curve joining $x$ and $x^{\prime}$ in $B$, we have

$$
\left|f(x)-f\left(x^{\prime}\right)\right| \leqq M(T) \sup _{y \in \gamma, i=1, \cdots, p}\left|a_{l}(y)-a_{l}\left(x^{\prime}\right)\right|^{1 / p} .
$$


$s^{\prime}(x)=M+1$ if $x \in D_{\alpha}-\Omega, s^{\prime}(x)=\max (s(x), M+1)$ if $x \in D_{\alpha} \cap \Omega$. Then, $s^{\prime}$ is pseudoconvex in $D_{\alpha}$ and, for any $r>0$, the set $\left\{x \in D_{\alpha} \mid s^{\prime}(x)<r\right\}$ has a closure in $D_{\alpha}$ which does not meet $\partial D \cap S$. Hence, the function $P(x)=(\alpha-p(x))^{-1}+s^{\prime}(x)$ is pseudoconvex in $D_{\alpha}$ and, for any $r>0$, we have $\left\{x \in D_{\alpha} \mid P(x)<r\right\} \subset \subset D_{\alpha}$. Hence, by Theorem $3, D_{\alpha}$ is Stein, which proves Theorem 4 in the special case when $X$ is irreducible.

To complete the proof of Theorem 4 in the general case, we have only to use the following theorem.

THEOREM 5. A complex space, all of whose irreducible components are Stein spaces is itself a Stein space.

This theorem is a special case of the theorem in [10], but since this is simpler to prove directly, we give a proof.

Proof of Theorem 5. Theorem 5 follows from the following result:

(5.3) Suppose that a complex space $X$ is the union of two closed analytic subsets $X_{1}, X_{2}$. If $X_{1}, X_{2}$ are Stein spaces, so is $X$.

In fact, suppose this proved and let $X$ be a complex space with the irreducible components $\left\{Y_{v}\right\}$, if $V_{k}=\bigcup_{v \leqq k} Y_{v}$, then $V_{k}$ is a Stein space. Let $f$ be a holomorphic function on $V_{k}$ and $E$ a discrete subset of $V_{k+1}, E \cap V_{k}=\varnothing$, and let $\phi$ be a holomorphic function on $E$ (i.e., to every point $x \in E$ is assigned a complex number $\phi(x))$. Since $V_{k+1}$ is Stein and $V_{k} \cup E$ an analytic set in $V_{k+1}$, there is a holomorphic function $g$ in $V_{k+1}$ such that $g\left|V_{k}=f, g\right| E=\phi$. Theorem 5 follows from this remark.

To prove (3.3), it is enough to show that for any coherent sheaf $\mathscr{T}$ of ideals on $X$, we have $H^{1}(X, \mathscr{T})=0$. Let $\mathcal{O}$ be the sheaf of germs of holomorphic functions on $X$ and $a_{v} \subset \mathcal{O}$ the subsheaf of germs of functions which vanish on $X_{v}$. Let $\mathcal{O}_{v}=\mathcal{O} / \mathfrak{a}_{v}$ and $\mathscr{T}_{v}=\mathscr{T} \otimes \mathcal{O}_{v}$, the tensor product being over $\mathcal{O}$. If $e_{v}$ is the image in $\mathcal{O}_{v}$ of the section 1 of $\mathcal{O}$, then any elemenc of $\left(\mathscr{T}_{v}\right)_{x}$ can be represented in the form $\alpha \otimes\left(e_{v}\right)_{x}$ where $\alpha \in \mathscr{T}_{x}$. Let $\eta: \mathscr{T} \rightarrow \mathscr{T}_{1} \oplus \mathscr{T}_{2}$ be the homomorphism defined by $\eta(\alpha)=\alpha \otimes e_{1} \oplus \alpha \otimes e_{2}$. Then $\eta$ is injective and if $\mathscr{H}$ is the cokernel of $\eta$, we have the exact sequence

$$
0 \rightarrow \mathscr{T}^{\eta} \stackrel{\mathscr{T}_{1}}{{ }} \mathscr{T}_{2} \stackrel{\rho}{\rightarrow} \mathscr{H} \rightarrow 0 .
$$

We assert that $\mathscr{H}$ has support contained in $Y=X_{1} \cap X_{2}$ and that $\mathscr{H}$ is a coherent $\mathcal{O}_{v}$ sheaf $(v=1,2)$. To prove the first statement, if $x \notin X_{v}$, then $\left(\mathbb{C}_{v}\right)_{x}=0$ so that $\eta$ is surjective at $x$, which shows that $\mathscr{H}_{x}=0$. To prove the second statement, it suffices to prove that $\mathfrak{a}_{v}$ annihilates $\mathscr{H}$ for $v=1,2$, i.e., that $\mathfrak{a}_{v} \cdot\left(\mathscr{T}_{1} \oplus \mathscr{T}_{2}\right)$ $\subset \eta(\mathscr{T})$. Now, $\mathfrak{a}_{v} \cdot \mathscr{T}_{v}=0$ and $\mathfrak{a}_{v} \cdot \mathscr{T}_{u}, v \neq \mu$, considered as a subsheaf of $\mathscr{T}_{1} \oplus \mathscr{T}_{2}$, is contained in $\eta(\mathscr{T})$, which proves that $\mathfrak{a}_{\boldsymbol{v}}$ annihilates $\mathscr{H}$.

Consider now the mapping

$$
\rho^{*}: H^{0}\left(X, \mathscr{T}_{1}\right) \oplus H^{0}\left(X, \mathscr{T}_{2}\right) \rightarrow H^{0}(X, \mathscr{H})
$$


induced by $\rho$. Now, $H^{0}\left(X, \mathscr{T}_{v}\right)=H^{0}\left(X_{v}, \mathscr{T}_{v}\right)$, and $H^{0}(X, \mathscr{H})=H^{0}\left(X_{v}, \mathscr{H}\right)$. Moreover the composition $\rho^{\prime}$ of the injection of $\mathscr{T}_{v}$ into $\mathscr{T}_{1} \oplus \mathscr{T}_{2}$ with $\rho$ is a surjective mapping of $\mathscr{T}_{v}$ onto $\mathscr{H}$, so that, since $X_{v}$ is Stein, $\rho^{\prime}$ induces a surjective mapping of $H^{0}\left(X_{v}, \mathscr{T}_{v}\right)$ onto $\mathrm{H}^{0}\left(X_{v}, \mathscr{H}\right)$. Hence the map $\rho^{*}$ is surjective.

Consider now the exact cohomology sequence associated to $\left(^{*}\right)$. We have the exact sequence

$$
\cdots \rightarrow H^{0}\left(X, \mathscr{T}_{1}\right) \oplus H^{0}\left(X, \mathscr{T}_{2}\right) \stackrel{\rho^{*}}{\rightarrow} H^{0}(X, \mathscr{H}) \rightarrow H^{1}(X, \mathscr{T}) \rightarrow 0
$$

since, the $X_{v}$ being Stein, we have $H^{1}\left(X, \mathscr{T}_{v}\right)=H^{1}\left(X_{v}, \mathscr{T}_{v}\right)=0$. But since $\rho^{*}$ is surjective, we deduce that $H^{1}(X, \mathscr{T})=0$, which proves (5.3).

Corollary 1 to Theorem 4. If $X$ is a Stein space with isolated singularities and $D$ an open set on $X$ which is locally Stein (i.e., any point of $\partial D$ has a neighborhood in $X$ whose intersection with $D$ is Stein), then D itself is a Stein space.

Corollary 2. If $X$ is any Stein space and $D$ is a locally Stein open subset of $X$ such that $D$ is strongly pseudoconvex at any point of $S$, the singular locus of $X$, then $D$ is a Stein space.

This follows from Theorem 4 since $\partial D \cap S$ has then a strongly convex neighborhood, and any strongly convex domain on a Stein space is Stein (see [9]).

The hypotheses of Corollary 2 are fulfilled in particular if $\partial D \cap S=\varnothing$.

REMARKS. (1) We can deduce from Corollary 1 that if $X$ is any two (complex) dimensional Stein space and $D$ a locally Stein open subset of $X$, then $D$ is Stein. In fact, it follows from Corollary 1 that if $\pi: Y \rightarrow X$ is the normalization of $X$, then $\pi^{-1}(D)$ is Stein. But $\pi: \pi^{-1}(D) \rightarrow D$ is the normalization of $D$, so that, by [10, Theorem 1], $D$ is Stein.

(2) We have not used the full force of our supposition that $X$ is a Stein space, and our proof gives for example the following theorem.

Let $X$ be a complex manifold on which global holomorphic functions give local coordinates at any point and suppose that any analytic subset of $X$ of codimension 1 can be defined by global equations. Then if $D$ is a relatively compact open subse $i$ of $X$ which is locally Stein, then $D$ is Stein.

6. $K$-complete manifolds: application of a vanishing theorem. Let $X$ be a complex manifold and $F$ a (holomorphic) line bundle on $X$. Suppose that $F$ is given by a covering $\left\{U_{i}\right\}$ and transition functions $f_{i j}: U_{i} \cap U_{j} \rightarrow C^{*}$. A (hermitian) metric on the fibres of $F$ is given locally by a form

$$
h(\xi, \eta)=h_{i}(x) \xi_{i} \bar{\eta}_{i}
$$

where $\xi_{i}, \eta_{i}$ are the fibre coordinates of the vectors $\xi, \eta$ over $U_{\imath}, h_{i}(x)>0$ being $C^{\infty}$ in $U_{i}$. In $U_{i} \cap U_{j}$, we have $h_{j}=\left|f_{i j}\right|^{2} h_{j i}$.

$F$ is called positive, if $h(\xi, \eta)$ can be so chosen that the hermitian form on $X$ associated to the alternate form 


$$
\chi=-\frac{1}{2 \pi i} \partial \cdot \bar{\partial} \log h_{i} \text { in } U_{i}
$$

is positive definite. Then $\chi$ defines a kähler metric on $X$. If $h(\xi, \eta)$ can be so chosen that the kähler metric defined by $\chi$ is complete, we say that $F$ is positive complete. (Note that the trivial bundle on $X$ is positive if and only if there exists a $C^{\infty}$ strongly pseudoconvex function on $X$.)

The following lemma is merely Proposition 11 of [2] formulated for the trivial bundle $F$.

LEMMA 8. Suppose that $X$ is a complex manifold on which there exists a strongly pseudoconvex function. If $K$ is the canonical bundle of $X$, suppose that $K^{-1}$ is positive complete. Then holomorphic functions on $X$ separate points and give local coordinates at each point of $X$.

To apply this, we make use of the following

LEMMA 9. Let $X$ be a complex manifold and $d s^{2}=\sum h_{\mu v} d z_{\mu} d \bar{z}_{v}$ be a hermitian form on $X$ such that the coefficients are "bounded" on any compact set. Suppose that $p$ is a $C^{\circ}$ strongly pseudoconvex function on $X$ such that the sets $\{x \in X \mid p(x) \leqq \alpha\}$ are compact. Then ihere is a $C^{\infty}$ function $\phi: R \rightarrow R$ such that if $u=\phi \circ p$, we have

$$
L(u)>d s^{2}
$$

Here, $L(u)$ denotes the Levi form

$$
\sum \frac{\partial^{2} u}{\partial z_{\mu} \partial \bar{z}_{v}} d z_{\mu} d \bar{z}_{v}
$$

Proof. We may suppose that $p>0$. Let $c_{v}>0$ be a constant such that $c_{v} L(p)>d s^{2}$ on the set of $x$ with $v \leqq p(x) \leqq v+1$. Let $h(t)$ be a $C^{\infty}$ function on $0 \leqq t<\infty$ such that $h(t)>c_{v}$ for $v \leqq t \leqq v+1, h^{\prime}(t)>0$. Define $\phi(t)=\int_{0}^{t} h(\tau) d \tau$, and put $u(x)=\phi(p(x))$. We have

$$
\begin{aligned}
L(u) & =\phi^{\prime}(p) L(p)+\phi^{\prime \prime}(p)|d p|^{2} \\
& =h(p) L(p)+h^{\prime}(p)|d p|^{2} \geqq h(p) L(p)>d s^{2} .
\end{aligned}
$$

The next lemma is a part of Theorem 1. However, we will use it for a proof of Theorem 5 below which is independent of the Heftungslemma.

LEMMA 8'. Let $X$ be a complex manifold and $p$ a $C^{\infty}$ strongly convex function on $X$ such that for any $\alpha>0$, the set $\{x \in X \mid p(X)<\alpha\} \subset \subset X$. Then, holomorphic functions on $X$ separate points and give local coordinates.

This follows at once from Lemma 8 and Lemma 9.

THEOREM 5. A holomorph-convex, K-complete manifold $X$ is a Stein manifold. 
Proof. Since $X$ is holomorph-convex, there is a sequence $\left\{\Delta_{r}\right\}$ of open sets in $X$, and holomorphic functions $f_{r, j}\left(j \leqq k_{r}\right)$ such that if we put $p_{r}^{\prime}(x)=\sum_{j \leqq k}\left|f_{r, j}\right|^{2}$, then $\Delta_{r}=\left\{x \in \Delta_{r+1} \mid p_{r}^{\prime}(x)<1\right\} ;$ moreover $\Delta_{r} \subset \subset \Delta_{r+1}, \bigcup \Delta_{r}=X$. Let $q$ be a $C^{\infty}$ strongly pseudoconvex function on $\Delta_{r+1}$ (this exists by Lemma 5). Then the function $p_{r}(x)=1 /\left(1-p_{r}^{\prime}(x)\right)+q(x)$ is a $C^{x}$ strongly pseudoconvex function in $\Delta_{r}$ and, for any $\alpha>0$, we have $\left\{x \in \Delta_{r} \mid p_{r}(x)<\alpha\right\} \subset \subset \Delta_{r}$. By Lemma $8^{\prime}$, holomorphic functions separate points and give local coordinates on $\Delta_{r}$. Clearly $\Delta_{r}$ is holomorph-convex, so that it is Stein. But now it is seen that $\Delta_{r}$ is $\Delta_{r+1}$-convex, so that holomorphic functions on $\Delta_{r}$ can be approximated by those on $\Delta_{r+1}$. It follows that holomorphic functions on $\Delta_{r}$ can be approximated by those on $X$. Hence holomorphic functions on $X$ separate points and give local coordinates. Hence $X$ is Stein.

We give two applications of Theorem 1. The proof that we give for the next theorem is very similar to that of Oka's theorem given in [9], but it is more natural in the context of covering manifolds.

THEOREM 6. Let $D$ be an open subset of $C^{n}$ which is Stein, and let $\tilde{D}$ be a covering manifold of $D$. Then $\tilde{D}$ is Stein.

Proof. Let $\pi: \tilde{D} \rightarrow D$ be the projection map; let $p$ be a $C^{\infty}$ strongly pseudoconvex function on $D$ such that for any $\alpha>0$, we have

$$
D_{\alpha}=\{x \in D \mid p(x)<\alpha\} \subset \subset D .
$$

Let $\tilde{p}=p \circ \pi$ and $\tilde{D}=\pi^{-1}\left(D_{\alpha}\right)$. Since $\tilde{p}$ is strongly convex and $\tilde{D}_{\alpha}=\{y \in \tilde{D} \mid$ $\tilde{p}(y)<\alpha\}$, we have only to prove that $\tilde{D}_{\alpha}$ is Stein.

Let $d s^{2}$ be a $C^{\infty}$ complete riemannian metric on $D$ and $d \tilde{s}^{2}=\pi^{*}\left(d s^{2}\right)$ the induced metric on $\tilde{D}$. Then $d \tilde{s}^{2}$ is complete in $\tilde{D}$ (see [13, p. 208]). Let $y_{0} \in \tilde{D}_{\alpha}$ and $\rho(y)$ the distance of $y \in \tilde{D}$ from $y_{0}$ with respect to $d \tilde{s}^{2}$. Let $|d z|^{2}$ be the Euclidean metric on $C^{\prime \prime}$. There is a constant $M>0$ so that

$$
\frac{1}{M}|d z|^{2}<d s^{2}<M|d z|^{2} \text { on } D_{\alpha+1} \text {. }
$$

This implies that if $y, y^{\prime} \in \tilde{D}_{\alpha}$ belong to an open connected set which is mapped isomorphically onto a convex set in $D_{\alpha+1}$ by $\pi$, then

$$
\left|\rho(y)-\rho\left(y^{\prime}\right)\right| \leqq M\left|\pi(y)-\pi\left(y^{\prime}\right)\right| \text {. }
$$

Let $\gamma \geqq 0$ be a $C^{\infty}$ function with compact support in the open ball $B_{\delta / 2}$ of radius $\delta / 2$ about $0 \in C^{n}$; we suppose that $\gamma(0)>0$. If $\delta$ is sufficiently small, for $y \in \tilde{D}_{\alpha}$, we can consider the open set $B_{\delta}(y)$ which is mapped isomorphically onto the ball of radius $\delta$ about $\pi(y)$. Then $\gamma$ defines a $C^{\infty}$ function $\gamma_{y}$ in $\boldsymbol{B}_{\delta}(y)$ if we put $\gamma_{y}\left(y^{\prime}\right)=\gamma\left(\pi(y)-\pi\left(y^{\prime}\right)\right)$. Define

$$
\rho_{1}(y)=\int_{B_{\delta}(y)} \gamma_{y}\left(y^{\prime}\right) \rho\left(y^{\prime}\right) d v_{y^{\prime}},
$$


where $d v_{y^{\prime}}$ is the volume element induced on $\tilde{D}$ by the Euclidean volume $d v$. Clearly $\left|\rho_{1}(y)-\rho(y)\right| \leqq M \int_{B_{\delta / 2}} \gamma(x) d v=C$. Moreover $\rho_{1}$ is a $C^{\infty}$ function in $\tilde{D}_{\alpha}$ and

$$
D_{\mu v} \rho_{1}=\frac{\partial^{2} \rho_{1}}{\partial y_{\mu} \partial \bar{y}_{v}}=\int_{B_{\delta}(y)} \frac{\partial \gamma_{y}\left(y^{\prime}\right)}{\partial y_{\mu}} \cdot \frac{\partial \rho\left(y^{\prime}\right)}{\partial \bar{y}_{v}^{\prime}} d v_{y^{\prime}}
$$

has absolute value $\leqq M \int_{B_{\delta / 2}}\left|\partial \gamma / \partial x_{\mu}\right| d v_{x}$, because of (1). Hence $\left|D_{\mu \nu} \rho_{1}\right|$ is bounded on $\tilde{D}_{\alpha}$. Hence there is a constant $K>0$ such that

$$
q(y)=\rho_{1}(y)+K|\pi(y)|^{2}
$$

is strongly pseudoconvex in $\widetilde{D}_{\alpha}$ and $q(y) \geqq \rho(y)-C$. Since $d \tilde{s}^{2}$ is complete, if $\beta<\alpha$, for any $r>0$ we have

$$
\left\{y \in \tilde{D}_{\beta} \mid q(y)<r\right\} \subset \subset \tilde{D}_{\alpha} .
$$

Hence $u(y)=(\alpha-\tilde{p}(y))^{-1}+q(y)$ is a $C^{\infty}$ strongly pseudoconvex function on $\tilde{D}_{\alpha}$ such that for $r>0,\left\{y \in \tilde{D}_{\alpha} \mid u(y)<r\right\} \subset \subset \tilde{D}_{\alpha}$. By Theorem 1, $\tilde{D}_{\alpha}$ is Stein.

RemarK. A method of K. Stein [15] enables one to deduce from Theorem 6 the theorem that any covering space of a Stein space is itself Stein. As a final application we prove

TheOReM 7. A holomorphic vector bundle $E$ over a Stein manifold $X$ is itself a Stein manifold.

Proof. Let $\pi: E \rightarrow X$ be the projection and $g_{i j}: U_{i} \cap U_{j} \rightarrow \mathrm{GL}(q, C)$ transition functions with respect to a covering $\left\{U_{i}\right\}$ defining $E$. Let $h_{i}$ be a $C^{\infty}$ map of $U_{i}$ into the space of positive definite hermitian matrices such that, on $U_{i} \cap U_{j}$, we have

$$
h_{j}={ }^{t} \bar{g}_{i j} h_{i} g_{i j}
$$

Let $\zeta^{(i)}$ be fibre coordinates on $\pi^{-1}\left(U_{i}\right)$. Define the $C^{\infty}$ function $\phi_{h}$ on $E$ by

$$
\phi_{h}(x, \zeta)=\zeta^{(i)} h_{i}(x) \zeta^{(i)} \text { if }(x, \zeta) \in \pi^{-1}\left(U_{i}\right) .
$$

Let $\Theta_{i}$ be the hermitian form associated to the alternate form $(1 / 2 \pi i) \bar{\partial}\left(h_{i}^{-1} \partial h_{i}\right)$. For differentials $d x, d \bar{x}$ at $x \in U_{i}$, define

$$
F_{h}(d x, d \bar{x}, \zeta)={ }^{i} \zeta^{(i)} h_{i}(x) \Theta_{i}(d x, d \bar{x}) \zeta^{(i)} .
$$

It is easy to verify (see e.g. [1, p. 257]) that if this hermitian form in $d x, d \bar{x}$ is positive definite for all $\zeta \neq 0$, then $\phi_{h}$ is strongly pseudoconvex outside the zero section of $E$.

We now consider a strongly pseudoconvex function $p$ on $X$ such that all sets $\{x \in X \mid p(x)<\alpha\}$ are relatively compact and consider the function

We have

$$
\phi=\phi_{h^{\prime}} \text { with } h_{i}^{\prime}=e^{p} \cdot h_{i} \text { in } U_{i} \text {. }
$$

$$
F_{h^{\prime}}=e^{p}\left\{L(p)^{i p(i)} h_{i} \zeta^{(i)}+{ }^{t} \zeta^{(i)} h_{i} \Theta_{i} \zeta^{(i)}\right\} .
$$


Now, since $h_{i}$ is positive definite, there is a constant $c_{i}>0$ corresponding to any $U_{i}^{\prime} \subset \subset U_{i}$ such that for ${ }^{t(i)} \zeta^{(i)}=1, \zeta^{(i)} h_{i} \zeta^{(i)} \geqq c_{i}$. By Lemma 8, we can choose $p$ such that $F_{h}$ is positive definite for any $\zeta^{(i)} \neq 0$. The function $\phi$ is then strongly pseudoconvex for $\zeta \neq 0$ and pseudoconvex everywhere in $E$. Consider now the function

$$
P(x, \zeta)=p \circ \pi+\phi
$$

on $E$. Clearly $P$ is strongly pseudoconvex everywhere on $E$ and, if $r>0$, we have $\{e \in E \mid P(e)<r\} \subset \subset E$. By Theorem $1, E$ is Stein.

REMARK. We can prove in the same way that any holomorphic vector bundle on any Stein space is Stein.

\title{
REFERENCES
}

1. A. Andreotti and H. Grauert, Théorèmes de finitude pour la cohomologie des espaces complexes, Bull. Soc. Math. France 90 (1962), 193-259.

2. A. Andreotti and E. Vesentini, Sopra un teorema di Kodaira, Ann. Scuola Norm. Sup. Pisa (3) 15 (1961), 283-309.

3. F. Docquier and H. Grauert, Levisches Problem und Rungescher Satz fur Teilgebiete Steinscher Mannigfaltigkeiten, Math. Ann. 140 (1960), 94-123.

4. H. Grauert, On Levi's problem and the imbedding of real-analytic manifolds, Ann. of Math. (2) 68 (1958), 460-472.

5. - Uber Modifikationen und exzeptionelle analytische Mengen, Math. Ann. 146 (1962), 331-368.

6. H. Grauert and R. Remmert, Komplexe Räume, Math. Ann. 136 (1958), 245-318.

7. S. Lokasiewicz, Sur le problème de la division, Studia Math. 18 (1959), 87-136.

8. R. Narasimhan, Imbedding of holomorphically complete complex spaces, Amer. J. Math. 82 (1960), 917-934.

9. - The Levi problem for complex spaces. II, Math. Ann. 146 (1962), 195-216.

10. - A note on Stein spaces and their normalisations, Ann. Scuola Norm. Sup. Pisa (3) 16 (1962), 327-333.

10a. - The Levi problem in the theory of functions of several complex variables, Proc. Internat. Congr. of Math., Stockholm, 1962.

11. K. Oka, Sur les fonctions analytiques de plusieurs variables complexes. IX. Domaines finis sans points critiques intérieur, Japan. J. Math. 23 (1953), 97-155.

12. R. Remmert, Holomorphe und meromorphe Abbildungen komplexer Räume, Math. Ann. 133 (1957), 328-370.

13. W. Rinow, Die innere Geometrie der metrischen Räume, Springer, Berlin, 1960.

14. J.-P. Serre, Quelques problemes globaux relatifs aux variétés de Stein, Colloque de Brussels, 1953.

15. K. Stein, Überlagerung holomorph-vollständiger komplexer Räume, Arch. Math. 7 (1956), 354-361.

16. G. Stolzenberg, Polynomially and rationally convex sets, Acta Math. 109 (1963), 259-289.

\author{
UNIVERSity OF Pisa, \\ Pisa, Italy \\ TATA INSTTTUTE OF FUNDAMENTAL RESEARCH, \\ BOMBAY, INDIA
}

